\title{
Label-Free Volumetric Quantitative Imaging of the Human Somatic Cell Division by Hyperspectral Coherent Anti-Stokes Raman Scattering
}

\author{
Arnica Karuna, ${ }^{\dagger, \|}$ Francesco Masia, ${ }^{\dagger}$ Marie Wiltshire, ${ }^{\ddagger}$ Rachel Errington, ${ }^{*}$, Paola Borri, ${ }^{\S}$ \\ and Wolfgang Langbein*, ${ }^{\dagger}$ \\ 'School of Physics and Astronomy, Cardiff University, The Parade, Cardiff CF24 3AA, United Kingdom \\ ${ }^{\ddagger}$ Division of Cancer and Genetics, School of Medicine, Cardiff University, Heath Park, Cardiff CF14 4XN, United Kingdom \\ ${ }^{\S}$ School of Biosciences, Cardiff University, Museum Avenue, Cardiff CF10 3AX, United Kingdom
}

Supporting Information

\begin{abstract}
Quantifying the chemical composition of unstained intact tissue and cellular samples with high spatiotemporal resolution in three dimensions would provide a step change in cell and tissue analytics critical to progress the field of cell biology. Label-free optical microscopy offers the required resolution and noninvasiveness, yet quantitative imaging with chemical specificity is a challenging endeavor. In this work, we show that hyperspectral coherent anti-Stokes Raman scattering (CARS) microscopy can be used to provide quantitative

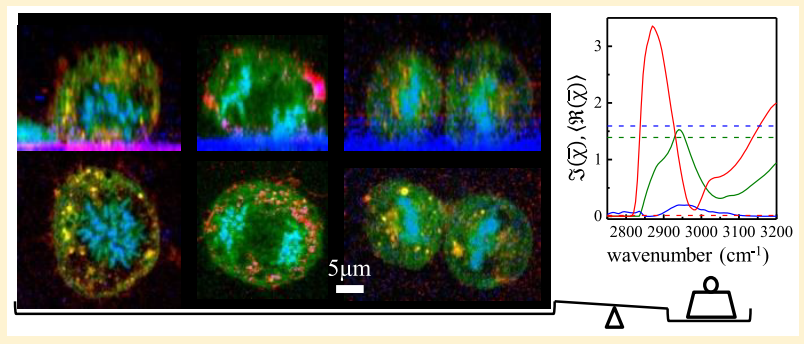
volumetric imaging of human osteosarcoma cells at various stages through cell division, a fundamental component of the cell cycle progress resulting in the segregation of cellular content to produce two progeny. We have developed and applied a quantitative data analysis method to produce volumetric threedimensional images of the chemical composition of the dividing cell in terms of water, proteins, DNAP (a mixture of proteins and DNA, similar to chromatin), and lipids. We then used these images to determine the dry masses of the corresponding organic components. The attribution of proteins and DNAP components was validated using specific well-characterized fluorescent probes, by comparison with correlative two-photon fluorescence microscopy of DNA and mitochondria. Furthermore, we map the same chemical components under perturbed conditions, employing a drug that interferes directly with cell division (Taxol), showing its influence on cell organization and the masses of proteins, DNAP, and lipids.
\end{abstract}

$\mathrm{O}$ ptical microscopy is the most used technique for imaging live and fixed cells and tissues with subcellular spatial resolution. Fluorescence based methods are typically employed, which generally require labeling to provide specificity, $^{1-3}$ and are widely utilized to study processes such as protein localization, interactions and cell metabolism. ${ }^{4}$ However, these techniques are innately invasive and can lead to fluorophore photobleaching and associated phototoxicity. Fluorescence staining also adds to the complexity of sample preparation, both for synthetic and genetically encoded fluorophores, and the potential for chemical/structural modifications of the sample undermines the experimental rationale of observing biological specimens in a nonperturbing way. Furthermore, the quantitative interpretation of fluorescence intensities in terms of the amount of labeled material is hindered by the complexity of the staining process and photobleaching.

As alternative to fluorescence microscopy, label-free chemical sensitivity and high spatial resolution are provided by Raman scattering microscopy, ${ }^{5}$ interrogating the vibrational resonances of endogenous molecules. However, spontaneous Raman scattering provides only weak signals, mandating long integration times and/or high excitation powers, limiting the applicability of the technique to imaging biological specimens. Specifically it is not suitable for live cells with cellular compartments that can move on a subsecond scale. Although recent developments toward light-sheet Fourier-transform Raman imaging ${ }^{6}$ have the potential to improve the acquisition speed, Raman microscopy is also affected by autofluorescence background, which limits its present applicability to volumetric imaging and makes it incompatible with fluorescent labeling.

Higher signals can be achieved in coherent Raman scattering (CRS), a nonlinear optical technique in which molecular vibrations are driven coherently by a light intensity modulation, typically achieved by the interference of two laser beams of different wavelengths. The signal enhancement in CRS originates from the coherent superposition of the Raman scattered fields, with a factor given by the number of vibrational modes in the focal volume, which can reach $10^{10}$ for pure materials such as lipids. ${ }^{7}$ CRS can be measured as

Received: October 13, 2018

Accepted: January 9, 2019

Published: January 9, 2019 
coherent anti-Stokes Raman scattering $(\mathrm{CARS})^{8}$ or stimulated Raman scattering (SRS), ${ }^{9}$ both having their advantages and drawbacks. ${ }^{7,10-12}$ CARS is generally easier to implement experimentally, since it occurs at a different wavelength compared to that of the exciting beams; thus, the signal can be easily separated via an optical bandpass filter and is blueshifted compared to the one-photon excited fluorescence background. On the other hand, it requires a more in depth data analysis, due to the contribution of vibrationally nonresonant backgrounds. Specifically, the CARS intensity can be described as $I_{\mathrm{C}} \propto|\chi|^{2}=\left|\chi_{\mathrm{R}}+\chi_{\mathrm{NR}}\right|^{2}$, where $\chi_{\mathrm{R}}\left(\chi_{\mathrm{NR}}\right)$ is the vibrationally resonant (nonresonant) component of the third-order susceptibility corresponding to the CARS process. $\chi_{\mathrm{R}}$ has both real and imaginary parts, while $\chi_{\mathrm{NR}}$ is due to an electronic contribution and is real for materials without twophoton absorption, which is the case for water, proteins, DNA, and lipids, for excitation pulses with wavelengths in the biological window, ${ }^{7} 700-1300 \mathrm{~nm}$. Therefore, one can rewrite $I_{\mathrm{C}} \propto\left|\chi_{\mathrm{R}}\right|^{2}+2 \mathcal{R}\left(\chi_{\mathrm{R}} \chi_{\mathrm{NR}}\right)+\left|\chi_{\mathrm{NR}}\right|^{2}$. The imaginary component of the resonant CARS susceptibility, i.e., $\mathcal{J}(\chi)=\mathcal{J}\left(\chi_{\mathrm{R}}\right)$ resembles the spontaneous Raman scattering cross-section, having peaks at the vibrational frequencies, and is linear in the concentration of the detected chemical species; therefore, it is the quantity of interest to be extracted from the measured CARS intensity. The challenge in retrieving $\mathcal{J}(\chi)$ from the CARS intensity is the determination of the phase of $\chi$. On the other hand, the presence of the nonresonant contribution is also an advantage, as it enhances the intensity of weak $\chi_{\mathrm{R}}$ by the homodyning in the interference term $2 \mathcal{R}\left(\chi_{\mathrm{R}} \chi_{\mathrm{NR}}\right)$, and it allows a nonresonant material to be used as reference for quantitative analysis. ${ }^{13}$ For the phase retrieval, effectively equivalent techniques ${ }^{14}$ in current use are based on a timedomain Kramers-Kronig (KK) formulation ${ }^{15}$ and the maximum entropy method. ${ }^{16}$ We have recently developed a phase-corrected KK approach (PCKK) which is improving on the KK formulation, by correcting not only the phase but also the amplitude of the signal, and results in a quantitative susceptibility in units of $\chi_{\text {ref }}$, relative to a known nonresonant reference material, $\bar{\chi}=\chi / \chi_{\text {ref. }}{ }^{17}$ Glass can be used as a reference material for frequencies above $1200 \mathrm{~cm}^{-1}$ and is convenient since it is typically part of the sample mount. For a larger frequency range, $\mathrm{NaCl}$ is a suitable choice as reference. ${ }^{13}$

Once the complex susceptibility is obtained, the resulting hyperspectral images still need to be analyzed in terms of the physical quantity of interest, which is the chemical composition of the specimen in terms of spatially resolved concentration maps of separated chemical components. Different techniques toward this goal have been proposed in literature, including classical least-squares analysis, ${ }^{18}$ independent component analysis, $^{19}$ and multivariate curve resolution analysis. ${ }^{20}$ We have developed an algorithm to analyze CARS hyperspectral images which determines quantitative spectra and absolute concentration images of separate chemical components without prior knowledge, called $\mathrm{FSC}^{3}$ (factorization into spectra and concentrations of chemical components). ${ }^{17,21}$ In this method the hyperspectral data are factorized as $\mathbf{D}=\sum_{i} \mathbf{C}_{i}$ $\times S_{i}+\mathbf{E}$, with $\mathbf{C}_{i}$ and $S_{i}$ being the non-negative concentration distribution and spectrum of the ith component, respectively, and $\mathbf{E}$ is the error, the norm of which is minimized. This method was recently used to quantitatively measure the lipid uptake in human adipose derived stem cells, ${ }^{22,23}$ to investigate the differentiation of stem cells, ${ }^{24}$ and toward high-throughput imaging using a Bessel beam and sparse sampling. ${ }^{25}$ It was also shown to be suited for the quantitative determination of the absolute volume of chemical components in three-dimensional imaging, using polystyrene beads as proof-of-principle. ${ }^{13}$ These studies opened the prospect of quantifying the volumetric chemical composition of intact unstained biological cells with submicron spatial resolution.

The cell cycle, a fundamental process underlying the proliferation of cell populations, broadly comprises two stages: interphase and cell division or mitosis. The cell division comprises various distinct stages in which the genetic material and organelles become segregated between the two progeny or daughter cells. ${ }^{26,27}$ As the cell progresses through these stages, the cell volume, ${ }^{28}$ chromatin density, ${ }^{29}$ and distribution/ number of organelles change. ${ }^{30,31}$ Quantitative mapping of these changes is key to the fundamental understanding of cell division and its monitoring in biomedically relevant applications. Traditionally, studies of the regulation and control of mitosis have been conducted using fluorescence labeling of cell organelles and small biomolecules. For instance, the Mitocheck consortium undertook phenotypic profiling using fluorescence timelapse microscopy to understand the critical genes responsible for cell division. ${ }^{32}$ Recently, label-free but chemically nonspecific quantitative phase contrast imaging was developed to study the change of the cell mass during the cell cycle. ${ }^{33-35}$

In the present paper, we demonstrate the application of volumetric quantitative hyperspectral CARS microscopy to image cells in three dimensions. We determine changes in the masses of cell components resembling proteins, a mixture of DNA and proteins, which we call here DNAP, and lipids at different stages of mitosis. As an outlook toward more biomedically relevant studies, we then apply this quantitative imaging to investigate the effect of an anticancer drug, Taxol, which stabilizes microtubule spindle structure and triggers the spindle-assembly checkpoint to halt the cells in metaphase. ${ }^{36}$

\section{MATERIALS AND METHODS}

In this study we used human osteosarcoma cells [U-2 OS] (ATCC HTB-96) transfected with a G2 M Cell Cycle Phase Marker (GE Healthcare, U.K.). The preparations were fixed, capturing a heterogeneous population transitioning through interphase and mitosis. Different sample preparations were produced to enable combined fluorescence and CARS modality for validation purposes and further to determine the consequences of drug perturbation. The two fluorescent molecular probes used have good two-photon cross-sections: (i) Mitotracker Orange diffuses across the plasma membrane of a live cell by passive transport and accumulates in the mitochondria $^{37}$ and (ii) DAPI (4',6-diamidino-2-phenylindole) binds to the minor groove of DNA, specifically the adenine and thymine rich regions of the DNA. ${ }^{38}$ Details on the sample preparation are given in the Supporting Information (SI), section 1 .

The data presented in this work were acquired on a homebuilt multimodal inverted microscope setup ${ }^{13,39}$ built around a Nikon Ti-U. Details are given in the SI, section 2. An example of DIC microscopy used to select cells for imaging with CARS is given in the SI, section 3. The hyperspectral CARS images were analyzed using the hyperspectral image analysis (HIA) software developed in our group, ${ }^{13,17,21}$ which retrieves the complex resonant susceptibility $\bar{\chi}$ relative to a glass reference. The volumetric images of $\bar{\chi}$ are then factorized into 


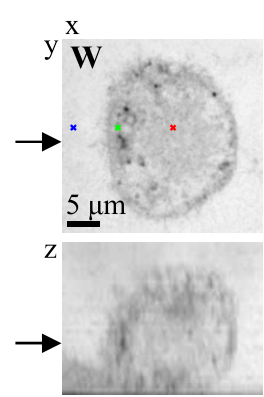

$1.084(0)$

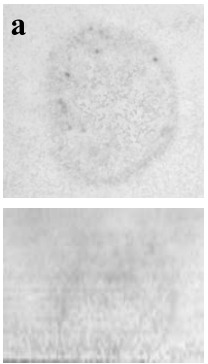

$1.106(0)$

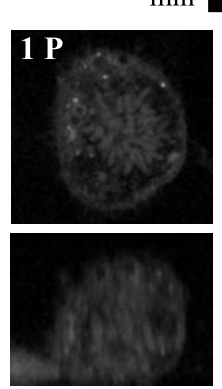

$0.496(0.179)$
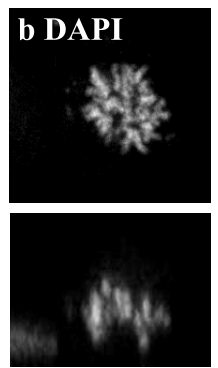

$1.8 \mathrm{MHz}$

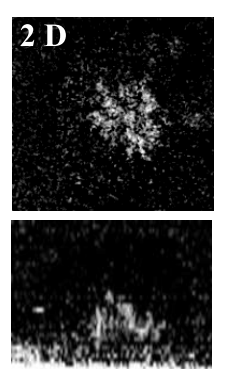

$0.116(0.007)$
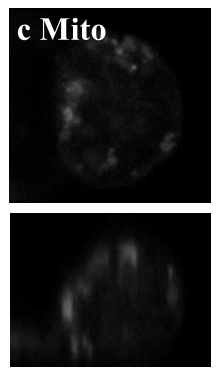

$13 \mathrm{MHz}$

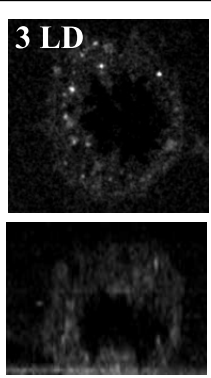

$0.077(0.029)$
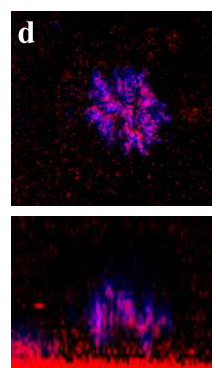

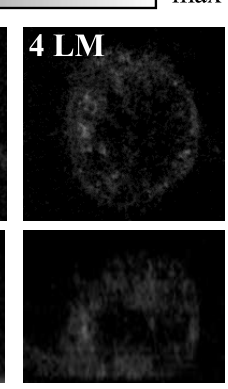

$0.711(0.025)$

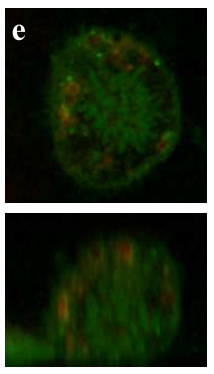

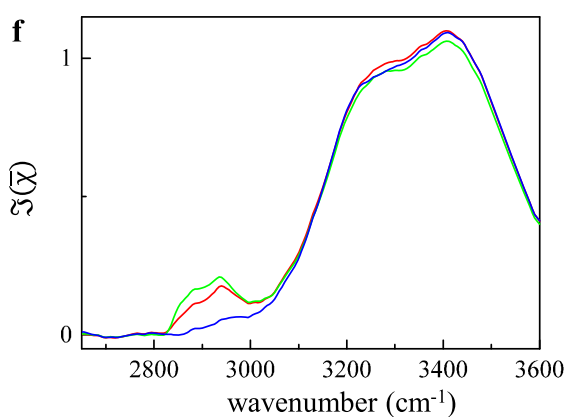

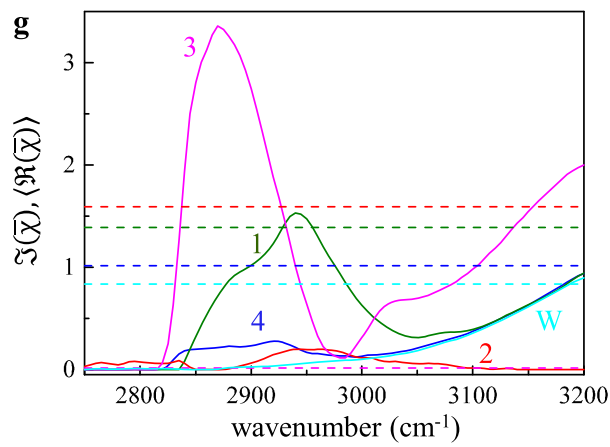

Figure 1. FSC F $^{3}$ concentrations and spectra of a U-2 OS cell in prometaphase. Images are $x y$ and $x z$ sections, given on a grayscale as shown from 0 to a maximum value indicated below each image. W, 1, 2, 3, 4 show the concentrations $C_{W, 1,2,3,4}$ of the respective components. Components 1 to 4 are also labeled $\mathrm{P}, \mathrm{D}, \mathrm{LD}$, and $\mathrm{LM}$, respectively, according to their chemical attribution. The corresponding maximum dry $\mathrm{v} / \mathrm{v}$ concentration is given in parenthesis. The arrows indicate the respective positions of the sections. Panel a shows the total concentration of water $C_{\mathrm{TW}}$. Panels $\mathrm{b}$ and $\mathrm{c}$ show the photoelectron rate in units of photoelectrons per second detected by the photomultiplier of TPF of DAPI and Mitotracker-orange, respectively. Panel $\mathrm{d}$ is a color overlay of $C_{2}$ in red and DAPI TPF in blue. Panel e shows $C_{1}$ in green and Mitotracker TPF in red. The PCKK spectra taken at the color coded points marked on $\mathrm{W}$ are given in panel $\mathrm{f}$. Panel $\mathrm{g}$ shows the spectra of the FSC ${ }^{3}$ components as labeled. Dashed lines show spectrally averaged $\mathcal{R}(\bar{\chi})$, and solid lines represent $\mathcal{J}(\bar{\chi})$. Corresponding $3 \mathrm{D}$ data are provided in the ref 40

susceptibilities and concentrations of chemical components via $\mathrm{FSC}^{3}$ which is using a non-negativity constrain on concentrations and the imaginary part $\mathcal{J}(\bar{\chi})$ as well as the spectrally averaged real part $\langle\mathcal{R}(\bar{\chi})\rangle$ of the component spectra. Unless otherwise specified, the CARS intensity data were taken over the spectral range $2600-3600 \mathrm{~cm}^{-1}$, and the susceptibility $\bar{\chi}$ was retrieved over this range and analyzed using $\operatorname{FSC}^{3}$ over the spectral range $2750-3200 \mathrm{~cm}^{-1}$. Above $3200 \mathrm{~cm}^{-1}, \bar{\chi}$ is dominated by the $\mathrm{OH}$ stretching vibrational resonances of water, which was excluded from the $\mathrm{FSC}^{3}$ analysis to reduce the influence of systematic errors in the dominating water component; note that the imaged volume contains about $95 \%$ water. For comparison, the $\mathrm{FSC}^{3}$ analysis of the cell in prometaphase over $2650-3600 \mathrm{~cm}^{-1}$ including the water resonance is shown in the SI, section 6i.

\section{RESULTS AND DISCUSSION}

CARS and TPF Imaging. We show here examples of U-2 OS cells in prometaphase, anaphase, and telophase. Figure 1 gives an overview of the $\mathrm{FSC}^{3}$ analysis for a cell in prometaphase (just before chromosome alignment). The factorization uses the full data containing $\sim 10^{6}$ voxels to retrieve components, resulting in well defined spectra with low noise, as shown in Figure 1g. For this analysis, we found that at least seven components in the $\mathrm{FSC}^{3}$ were required (see the Materials and Methods section and the SI). The components shown resemble protein $\left(C_{1} \equiv C_{\mathrm{P}}\right)$, DNAP $\left(C_{2} \equiv C_{\mathrm{D}}\right)$, and lipids split into lipid droplets $\left(C_{3} \equiv C_{\mathrm{LD}}\right)$ and lipid membranes $\left(C_{4} \equiv C_{\mathrm{LM}}\right)$, attributed according to their spatio-spectral profiles. The other components are water $\left(C_{5}\right.$ and $\left.C_{6}\right)$ and glass $\left(C_{7}\right.$; see the SI, Figure S21). We note that due to spectral similarities and low concentration of nonlipidic organic material in the cells, we do not obtain pure components for protein and DNA. On the other hand, lipid spectra are better distinguished, identified by their abundance of $\mathrm{C}-\mathrm{H}$ bonds. Furthermore, lipids, if not part of membrane structures, are segregated into lipid droplets and thus form localized, highly concentrated chemical species which can be identified readily in $\mathrm{FSC}^{3}$.

Considering the lipid components, we observe that $C_{\mathrm{LM}}$ is localized at the cell membrane, having a smaller $\mathcal{J}(\bar{\chi})$ than that of $C_{\mathrm{LD}}$, which is localized in lipid droplets, with a weak contribution at the cell boundary. $C_{\mathrm{D}}$ is located in the chromosomal region, and $C_{\mathrm{P}}$ is distributed across the cell, including the chromosomal region where it shows some colocalization with $C_{\mathrm{D}}$. This is not surprising, as both components contain protein. Note that in this paper we refer to the region containing the DNA as the chromosomal region, as during mitosis the nuclear compartment is not present. In addition to the $\mathrm{FSC}^{3}$ components, we define a sum water component $C_{\mathrm{W}}$ (Figure $1 \mathrm{~W}$ ) by combining the water components $C_{5}$ and $C_{6}$ with a concentration given by the sum of the concentrations and a spectrum given by the average of the spectra weighted by their mean concentration over the imaged volume. Furthermore, we show in Figure 1a the total concentration of water $C_{\mathrm{TW}}=\sum C_{i} \gamma_{i}^{\mathrm{w}}$, calculated by adding all of the component concentrations weighted with their water fractions $\gamma_{i}^{\mathrm{w}}$. The latter is deduced from the dry fractions $\gamma_{i}^{\mathrm{d}}$ as 
$\gamma_{i}^{\mathrm{w}}=1-\gamma_{i}^{\mathrm{d}}$. The dry fractions $\gamma_{i}^{\mathrm{d}}$ are calculated as described later and are given in Table 1 . We find $\gamma_{1}^{\mathrm{w}}=0.639, \gamma_{2}^{\mathrm{w}}=0.942$,

Table 1. Volumes and Masses of $C_{1}, C_{2}, C_{3}$, and $C_{4}$ of Labelled U-2 OS Cells in Various Stages of Mitosis, over the Chromosomal and Cell Volumes ${ }^{a}$

\begin{tabular}{|c|c|c|c|c|c|}
\hline$i$ & type & $\gamma_{i}^{\mathrm{d}}$ & $V_{i}\left(\mu \mathrm{m}^{3}\right)$ & $V_{i} / V_{\mathrm{ROI}}(\%)$ & $M_{i}(\mathrm{pg})$ \\
\hline \multicolumn{6}{|c|}{ Figure 1 - prometaphase - chromosomal volume - $982 \mu \mathrm{m}^{3}(20 \%)$} \\
\hline 1 & $\mathrm{P}$ & 0.361 & $32.5 \pm 0.3$ & 3.31 & $42.3(43.2)$ \\
\hline 2 & $\mathrm{D}$ & 0.058 & $1.2 \pm 0.6$ & 0.13 & $1.9(0.9)$ \\
\hline 3 & $\mathrm{LD}$ & 0.377 & $1.81 \pm 0.05$ & 0.18 & 1.6 \\
\hline 4 & LM & 0.035 & $1.8 \pm 0.1$ & 0.18 & 1.6 \\
\hline \multicolumn{6}{|c|}{ Figure 1 - prometaphase - cell volume $-4944 \mu \mathrm{m}^{3}$} \\
\hline 1 & $\mathrm{P}$ & 0.361 & $133 \pm 1$ & 2.70 & $173.5(177.3)$ \\
\hline 2 & $\mathrm{D}$ & 0.058 & $3.1 \pm 0.6$ & 0.06 & $4.7(0.9)$ \\
\hline 3 & $\mathrm{LD}$ & 0.377 & $17.8 \pm 0.5$ & 0.36 & 16.0 \\
\hline 4 & LM & 0.035 & $11.2 \pm 0.2$ & 0.23 & 10.0 \\
\hline \multicolumn{6}{|c|}{ Figure 2 - anaphase - chromosomal volume - $675 \mu \mathrm{m}^{3}(14 \%)$} \\
\hline 1 & $\mathrm{P}$ & 0.361 & $17.6 \pm 0.2$ & 2.61 & $22.9(24.7)$ \\
\hline 2 & $\mathrm{D}$ & 0.058 & $1.1 \pm 0.6$ & 0.17 & $1.7(-0.1)$ \\
\hline 3 & LD & 0.377 & $0.84 \pm 0.02$ & 0.12 & 0.8 \\
\hline 4 & LM & 0.035 & $6.2 \pm 0.1$ & 0.91 & 5.5 \\
\hline \multicolumn{6}{|c|}{ Figure 2 - anaphase - cell volume - $4788 \mu \mathrm{m}^{3}$} \\
\hline 1 & $\mathrm{P}$ & 0.361 & $74.4 \pm 0.7$ & 1.55 & $96.7(104.3)$ \\
\hline 2 & $\mathrm{D}$ & 0.058 & $5.0 \pm 0.6$ & 0.11 & $7.5(-0.1)$ \\
\hline 3 & LD & 0.377 & $12.2 \pm 0.3$ & 0.25 & 10.9 \\
\hline 4 & $\mathrm{LM}$ & 0.035 & $42.3 \pm 0.6$ & 0.88 & 37.8 \\
\hline \multicolumn{6}{|c|}{ Figure 3 - telophase - chromosomal volume $*-317 \mu \mathrm{m}^{3}(11 \%)$} \\
\hline 1 & $\mathrm{P}$ & 0.361 & $18.1 \pm 0.2$ & 5.72 & $23.6(24.3)$ \\
\hline 2 & $\mathrm{D}$ & 0.058 & $0.4 \pm 0.6$ & 0.13 & $0.6(-0.04)$ \\
\hline 3 & LD & 0.377 & $1.04 \pm 0.03$ & 0.33 & 0.9 \\
\hline 4 & LM & 0.035 & $0.04 \pm 0.1$ & 0.01 & 0.04 \\
\hline \multicolumn{6}{|c|}{ Figure 3 - telophase - cell volume* $-2802 \mu \mathrm{m}^{3}$} \\
\hline 1 & $\mathrm{P}$ & 0.361 & $87.5 \pm 0.9$ & 3.12 & $113.7(117.0)$ \\
\hline 2 & $\mathrm{D}$ & 0.058 & $2.1 \pm 0.6$ & 0.08 & $3.2(-0.04)$ \\
\hline 3 & LD & 0.377 & $17.1 \pm 0.5$ & 0.61 & 15.3 \\
\hline 4 & LM & 0.035 & $0.5 \pm 0.1$ & 0.02 & 0.5 \\
\hline
\end{tabular}

${ }^{a}$ The percentage of chromosomal to cell volume is indicated. The telophase results are per cell. The rebalanced masses $\tilde{M}_{\mathrm{P}}$ and $\tilde{M}_{\mathrm{D}}$ are given in parentheses.

$\gamma_{3}^{\mathrm{w}}=0.623, \gamma_{4}^{\mathrm{w}}=0.965, \gamma_{5}^{\mathrm{w}}=\gamma_{6}^{\mathrm{w}}=1.00$, and $\gamma_{7}^{\mathrm{w}}=0 . C_{\mathrm{TW}}$ shows a homogeneous distribution across the cell close to unity, with a slightly negative contrast in the regions corresponding to the lipid and DNAP components, having a concentration of dry mass within the PSF up to about $20 \%$. The retrieved spectra, at the three points color coded in the $C_{\mathrm{W}}$ map, are given in Figure If and reveal small spectral differences, in the range of 0.1 in $\mathcal{J}(\bar{\chi})$. This is much smaller than $\mathcal{J}(\bar{\chi})$ of bulk lipid having a peak value ${ }^{17}$ of about 6 , reflecting the small concentration of organic material in the focal volume. 3D data in the form of movies for the concentration maps of the chemical components are available online (see data in ref 40).

Results from TPF microscopy and their comparison with the FSC $^{3}$ analysis of CARS images is shown in Figure $1 \mathrm{~b}-\mathrm{e}$, and their correlation is analyzed in the SI, section 5. Good colocalization of $C_{\mathrm{D}}$ (red) with DAPI TPF (blue) is observed in Figure $1 \mathrm{~d} . C_{\mathrm{P}}$ (green) and Mitotracker-orange TPF (red) shown in Figure 1e are not significantly colocalized. $C_{\mathrm{LM}}$ indicates some colocalization with the Mitotracker-TPF, supporting the attribution of $C_{\mathrm{LM}}$ to lipid membranes which are dense in mitochondria. This finding suggests that mitochondria are detected in $\mathrm{FSC}^{3}$ not as a separated chemical component but as part of the protein $C_{\mathrm{P}}$ and lipid membrane $C_{\mathrm{LM}}$ components.

Figure 2 shows the volumetric imaging of a cell in anaphase, which has been analyzed with FSC $^{3}$ together with the cell in prometaphase shown in Figure 1. The genetic material $\left(C_{\mathrm{D}}\right)$ is distributed at the two poles of the oval shaped cell (visible in the concentration map of $C_{\mathrm{p}}$ ). These are characteristic features of cells in anaphase. $C_{\mathrm{LD}}$ shows small lipid droplets alongside an extracellular and membrane background, while $C_{\mathrm{LM}}$ appears with a cell background also showing some lipid aggregates/ droplets. Furthermore, we observe only a partial polarization of the lipid components which appear to be concentrated in the top half of the cell, indicating their nondesignation to either of the future daughter cells. We note good correlation between $C_{\mathrm{D}}$ and DAPI TPF, while $C_{\mathrm{P}}$ and Mitotracker TPF show a smaller degree of colocalization, consistent with the findings for the cell in prometaphase.

In Figure 3, the volumetric imaging of two future daughter cells in telophase is shown, analyzed together with the cells in prometaphase and anaphase (i.e., all retrieved component spectra are in common). We note a slightly asymmetric cytoplasmic cleavage along the vertical $(y)$ direction. Furthermore, the chromosomal material is split into two parts, as shown by $C_{\mathrm{D}}$. The nuclear membranes are still lacking, typical of cells in telophase. The lipid droplets are clearly visible in $C_{\mathrm{LD}}$, with a general background in the imaged volume. We note that $C_{\mathrm{LM}}$ has a negligible concentration in

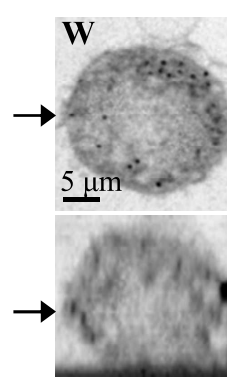

$1.085(0)$

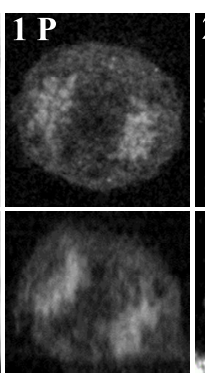

$0.191(0.068)$

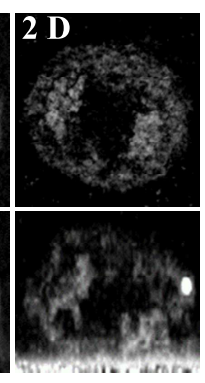

$0.102(0.005)$

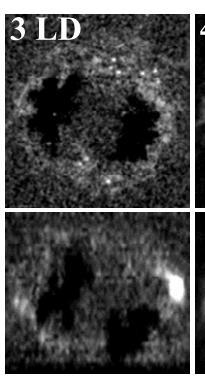

$0.028(0.010)$

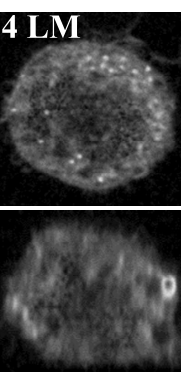

$0.852(0.030)$

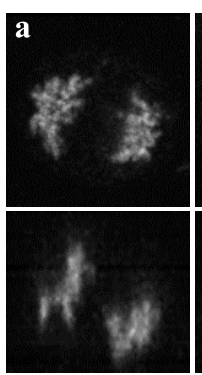

$1.4 \mathrm{MHz}$
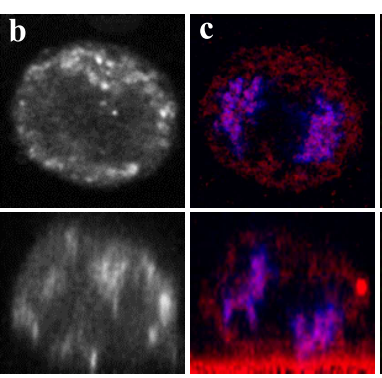

$4.5 \mathrm{MHz}$

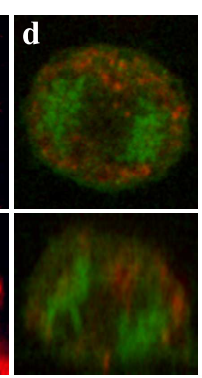

Figure 2. FSC ${ }^{3}$ analysis and TPF imaging of a U-2 OS cell in anaphase. Images are $x y$ and $x z$ sections. W, 1, 2, 3, 4 show concentrations $C_{W, 1,2,3,4}$ of the respective components with spectra as shown in Figure $1 \mathrm{~g}$ on a grayscale as in Figure 1. Panels a and $\mathrm{b}$ show the detected photoelectron rate of TPF of DAPI and of Mitotracker-orange, repectively. Panel $\mathrm{c}$ is a color overlay of $C_{2}$ in red and DAPI TPF in blue. Panel $\mathrm{d}$ is a color overlay of $C_{1}$ in green and Mitotracker TPF in red. 


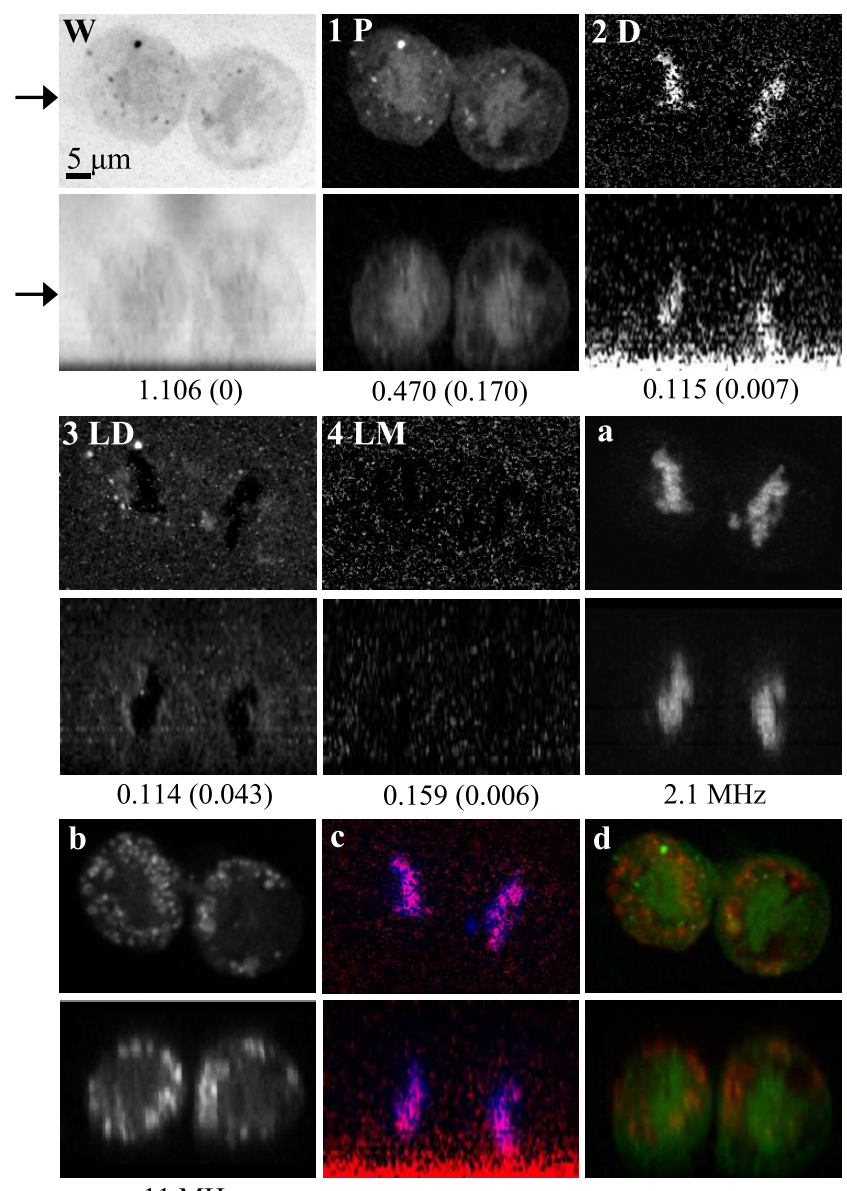

$11 \mathrm{MHz}$

Figure 3. As in Figure 2 but for a cell pair in telophase. This data was acquired over the IFD range $(2600-3800) \mathrm{cm}^{-1}$ with step size 6 $\mathrm{cm}^{-1}$.

these cells. As observed for the cells in prometaphase and anaphase, the concentration distribution of $C_{\mathrm{D}}$ is correlated with DAPI TPF, and $C_{\mathrm{P}}$ and $C_{\mathrm{LM}}$ are correlated with Mitotracker TPF. The FSC ${ }^{3}$ components not shown in Figures 2 and 3 are given in the SI, in Figures S22 and Figure S23, respectively.

Volume and Mass Analysis. The quantitative capability of hyperspectral CARS imaging analyzed with FSC $^{3}$ was demonstrated using beads of pure material. ${ }^{13}$ Here, we use this capability to quantify the volumes and dry masses of individual chemical components of cells in mitosis. Notably, this analysis is challenging due to the small volume fraction of organic material in the cells.

Calculation of Dry Fractions. The $\mathrm{FSC}^{3}$ components typically contain a significant fraction of water, as can be seen in their spectra (Figure 1g), showing a rising $\mathcal{J}(\bar{\chi})$ in the $3100-3200 \mathrm{~cm}^{-1}$ range. This is expected, given that these components are used to describe data with a maximum dry concentration over the PSF well below unity. Therefore, in order to quantify the dry masses of the various components, we have to remove the water contribution. For IFDs above 3100 $\mathrm{cm}^{-1}$ we can assume that $\mathcal{J}(\bar{\chi})$ is dominated by the water component, while the organic components have significant $\mathcal{J}(\bar{\chi})$ in the range $2800-3100 \mathrm{~cm}^{-1}$.

We remove the contribution of water to the spectra by subtracting a fraction $\alpha$ of the water spectrum $\mathcal{J}\left(\bar{\chi}_{\mathrm{W}}\right)$, resulting in the dry spectrum $\mathcal{J}\left(\bar{\chi}_{\text {dry }}\right)=\mathcal{J}(\bar{\chi})-\alpha \mathcal{J}\left(\bar{\chi}_{\mathrm{W}}\right)$, where $\alpha$ is chosen to result in $\mathcal{J}\left(\bar{\chi}_{\text {dry }}\right)$ of zero at the upper limit of the IFD range analyzed by FSC ${ }^{3}$, where $\mathcal{J}\left(\bar{\chi}_{\mathrm{W}}\right)$ is highest. The values of $\alpha$ and the corresponding spectra are given in the SI, section 9.

The dry volume fraction $\gamma_{i}^{\mathrm{d}}$ of an $\mathrm{FSC}^{3}$ component $C_{i}$ is then taken as the ratio of the integral $A_{i}$ of $\mathcal{J}\left(\bar{\chi}_{\text {dry }}\right)$ over the $\mathrm{CH}$ stretch region to the corresponding integral $A_{\mathrm{dry}}$ of the dry bulk material of the component. The latter is estimated as $A_{\text {dry }}=$ $A_{\mathrm{OA}} F_{i}$ using the integral $A_{\mathrm{OA}}$ of a measured pure dry material which is available, here oleic acid (OA). The factor $F_{i}$ is calculated assuming that the integral is proportional to the volume density of $-\mathrm{H}$ bonds in the material with vibrational frequencies in the integration range. This factor can therefore be expressed as

$$
F_{i}=\frac{b_{i}}{b_{\mathrm{OA}}} \frac{m_{\mathrm{OA}}}{m_{i}} \frac{\rho_{i}}{\rho_{\mathrm{OA}}}
$$

where $b_{\mathrm{OA}}\left(b_{i}\right)$ is the number of Raman active bonds in a molecule of OA $\left(C_{i}\right)$ in the IFD range considered, $m_{\mathrm{OA}}\left(m_{i}\right)$ is the molecular weight of $\mathrm{OA}\left(C_{i}\right)$ and $\rho_{\mathrm{OA}}\left(\rho_{i}\right)$ is the mass density of $\mathrm{OA}\left(C_{i}\right)$, respectively. The corresponding values are given in the SI, in Table S2 for protein, DNA, and common lipids in cells, palmitic acid (PA) and stearic acid (SA), ${ }^{41}$ as well as OA. In the calculation of these factors, for protein, the relative abundance of twenty amino acids ${ }^{42}$ which make up proteins was taken into account. DNAP, using chromatin as a model, was considered to be a mixture of protein and DNA in a 2:1 ratio. $^{43}$ Furthermore, the four constituent bases of DNA were assumed to occur in equal numbers.

For lipids, $F_{3}=F_{4}=1.0$ was calculated using an average of the OA, PA, and SA densities, ${ }^{4-46}$ which is equal to the density of oleic acid, $\rho_{\mathrm{OA}}=0.895 \mathrm{~g} / \mathrm{cm}^{3}$. For bulk OA, the integral $A_{\mathrm{OA}}=797 \mathrm{~cm}^{-1}$ of $\mathcal{J}(\bar{\chi})$ over the IFD range 2650$3100 \mathrm{~cm}^{-1}$ was measured. We calculate the density of DNAP as $1.5 \mathrm{~g} / \mathrm{cm}^{3}$ using the protein density, $1.3 \mathrm{~g} / \mathrm{cm}^{3}$, and the DNA density, ${ }^{47} 2 \mathrm{~g} / \mathrm{cm}^{3}$. For protein $\left(C_{1}\right)$ we find $F_{1}=0.58$. For DNA, this factor is 0.53 , so that for DNAP, $F_{2}=0.56$ is calculated using a weighted average of factors for protein and DNA in a 2:1 ratio. We note that the $F_{i}$ for protein and DNAP are about half that of lipid, reflecting the lower abundance of hydrogen in these materials. Further discussion of the accuracy of this procedure is given later.

Volumes of the Dry Components. Volume calculations to estimate the mass of the organic $\mathrm{FSC}^{3}$ components in the cells were made in two different regions of interest (ROI): the chromosomal region and the whole cell. To define the chromosomal ROI, we thresholded the corresponding TPFDAPI images, and the cell ROI was determined using the cell perimeter visible in $C_{\mathrm{P}}$. The dry volumes $V_{i}$ and masses $M_{i}$ of the FSC $^{3}$ components $C_{i}$ were then calculated as volume integrals

$$
V_{i}=\gamma_{i}^{d} \int_{\mathrm{ROI}} C_{i}(\mathrm{r}) \mathrm{d} V \text { and } M_{i}=V_{i} \rho_{i}
$$

with the three-dimensional position $\mathbf{r}$. The results of these volumetric calculations over the chromosomal and cell ROIs are given in Table 1 . The errors given for $V_{i}$ are accounting for the error in $A_{i}$ due to the selection of $\alpha$ and the fluctuations in the spectra. The dry volumes reported for the cells in telophase 


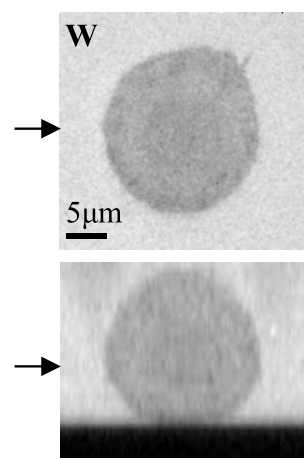

$1.291(0)$
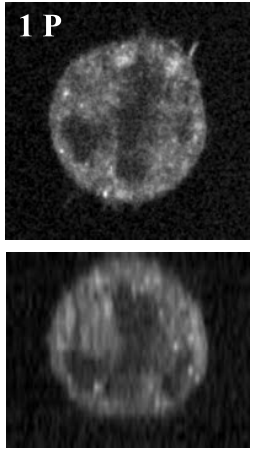

$0.408(0.127)$

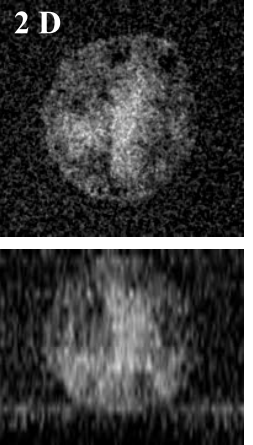

$0.196(0.025)$

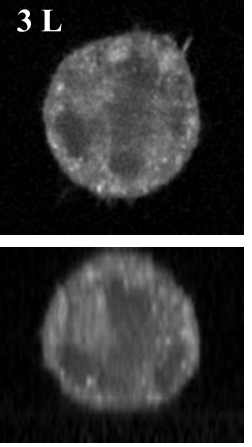

$0.090(0.068)$

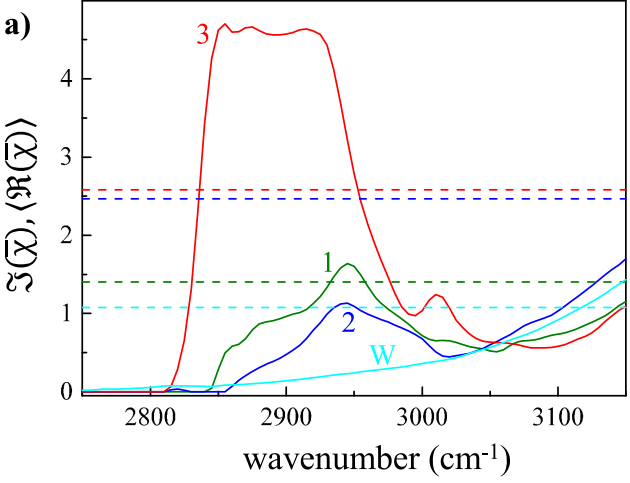

Figure 4. FSC 3 analysis of a Taxol treated U-2 OS cell. Images W, 1, 2, 3 show $x y$ and $x z$ sections of concentrations of the components $C_{W, 1,2,3}$ with spectra given in panel a. Grayscales are as in Figure 1. This data was acquired with $z$ step size of $1 \mu \mathrm{m}$ and an IFD range of $2600-3800 \mathrm{~cm}^{-1}$.

are given as average per daughter cell. From here onward, we will indicate such reference by an asterix * for all fields of view comprising more than one cell.

The results in Table 1 show cell dry masses in the 100-200 pg range. Most of this is protein, supplemented by about $10 \%$ lipids in droplets in $C_{\mathrm{LD}}$ and a similar amount of lipids in membranes in $C_{\mathrm{LM}}$. Over the chromosomal volumes, we observe, as expected, low levels of lipids. Additionally, the composition of the chromosomal region is dominated by the protein component $C_{\mathrm{P}}$. The DNAP component $C_{\mathrm{D}}$ amounts to a few percent of the cell dry mass, about $1.8 \mathrm{pg}$ in prometaphase and anaphase and $0.6 \mathrm{pg}$ after division in telophase. We would expect the chromosomal content to split in equal parts into the two daughter cells and, therefore, expect a mass of $0.9 \mathrm{pg}$ in telophase, which is consistent with the measurements within error. However, these DNAP masses are at the limit of detection (see error values) and significantly lower than the known DNA content of a diploid cell, which is 5-7 pg before and 10-14 pg after the $S$ phase. ${ }^{43}$ We note that, if DNAP is assigned to pure DNA, the mass would increase by a factor of 1.37 due to the higher density. Furthermore, due to the spectral similarities of DNA with protein, which has a higher concentration in the cell, some of the DNA could be contributing to $C_{\mathrm{P}}$. Therefore, we examine the sum of the masses of protein and DNAP components in the chromosomal region, which is about $44.2 \mathrm{pg}$ for the prometaphase, $24.6 \mathrm{pg}$ for the anaphase, and $24.2 \mathrm{pg}$ for each of the telophase cells. This is in good agreement with the expectation that the mass of chromatin in mitotic cells (with double the DNA content compared to a diploid G1 cell) would vary between 30 and 42 $\mathrm{pg}$, considering 10 to $14 \mathrm{pg}$ of DNA per mitotic cell, and the mass ratio of protein:DNA $(2: 1)$ in chromatin. The factorization of the cell composition into only few components thus provides masses corresponding to protein and DNAP within the chromosomal region in all three cells, in agreement within error with the literature values of chromatin mass in a cell. With respect to the lipid composition of the cell, we observe most of the lipids $\left(C_{\mathrm{LD}}\right.$ and $\left.C_{\mathrm{LM}}\right)$ outside of the chromosomal region.

In an attempt to better separate the mass of DNA from proteins, we can use the prior knowledge that the DNA mass outside of the nuclear region is zero. We define accordingly rebalanced masses of protein, $\tilde{M}_{\mathrm{P}}=M_{\mathrm{P}}+\beta M_{\mathrm{P}}$ and DNAP, $\mathcal{M}_{\mathrm{D}}$ $=M_{\mathrm{D}}-\beta M_{\mathrm{P}}$, where $\beta=M_{\mathrm{D}} / M_{\mathrm{P}}$ with the DNAP mass $M_{\mathrm{D}}$ and protein mass $M_{\mathrm{P}}$ in the extra-chromosomal region, so that $\tilde{M}_{\mathrm{D}}$ $=0$ in this region, as required. The resulting rebalanced masses are given in parentheses in Table 1 . We observe, for this factorization, a $\tilde{M}_{\mathrm{D}}$ of zero within error in anaphase and telophase cells, showing that DNA is not fully separated from other protein components in the present data and also that the DNA mass is close to the detection limit. It is also possible that the determination of the DNA mass is affected by the DAPI staining, since, as will be shown later, for the unlabeled drugged cells the rebalanced masses are in agreement with the expected DNA mass.

An interesting feature of cell division is chromosome compaction, which is still not fully explained. A major challenge in these studies is related to the use of fluorophores and the associated analysis with variable results depending on the specific measurement and analysis methods used. ${ }^{29}$ CARS can contribute results to such studies because of its noninvasive high resolution chemical specificity. Considering the volume ROIs, we observe that the ratio of chromosomal to cell volume decreases from prometaphase (20\%) to anaphase (14\%) to telophase cells (11\%), indicating progressing chromatin compaction. At the same time, the ratio of chromosomal to cell protein and DNAP masses changes much less from $25 \%$, to $24 \%$, to $21 \%$, quantifying the chromosome compaction.

The reproducibility of the analysis against random initial guesses for spectra and concentrations in the $\mathrm{FSC}^{3}$ factorization is discussed in the SI, section 7 through an alternative simultaneous factorization of the three data sets. We find that mass and volume for the dominant protein component have a relative systematic error of around $10 \%$. Changes are more significant for chemical species with small dry fractions and low signal-to-noise ratios such as DNAP. To investigate the reproducibility for cells of equal stage, we have analyzed a second prometaphase cell (see the SI, Figure S10), showing consistent results. The factorization can be guided to provide more reproducibility, as will be used below. Further discussion of systematic errors in dry mass determination is given in the SI, section 10 .

U-2 OS Cells Treated with the Anticancer Drug Taxol. The ability of an imaging technique to quantify changes in the intracellular chemical composition following drug treatment is of great importance to understand the effects of drugs at a subcellular level and to assess the treatment consequences (pharmacodynamics). With this goal in mind, we explore here the application of quantitative CARS microscopy to cells treated with the anticancer drug Taxol, which promotes polymerization of tubulin, thereby causing an arrest in 
metaphase which usually downstream leads to apoptosis. ${ }^{48}$ At low concentrations, Taxol interferes with microtubule dynamics and prevents the attachment of kinetochores to the spindle apparatus resulting in cell cycle arrest in metaphase by activation of the spindle assembly checkpoint. ${ }^{49}$ We performed hyperspectral CARS measurements and $\mathrm{FSC}^{3}$ analysis on Taxol treated U-2 OS cells. To promote reproducibility of the factorization, we took a systematic approach where a guided FSC $^{3}$ was used in which a set of voxels with a priori chosen spectra are added to the data to form guides which "suggest" to the factorization algorithm the component spectra while not constraining it. The weights $w$ of the guide spectra define the relative volume of the guides with respect to the data being analyzed. Three guide spectra (SI, Figure S15) were obtained by factorizing the composition of a control cell (SI, Figure S16). We also lowered the upper limit of the IFD range to $3150 \mathrm{~cm}^{-1}$ to reduce the influence of the water in the factorization from which these guides were obtained.

Two Taxol treated cells were factorized simultaneously using the IFD range $(2750-3150) \mathrm{cm}^{-1}$ and $w \approx 4 \%$ for each of the guide spectra. One of the cells in this analysis is shown in Figure 4. $C_{1} \equiv C_{\mathrm{P}}$, representing proteins, is homogeneous over the cell, with slightly negative contrast in the nuclear region. In $C_{2} \equiv C_{\mathrm{D}}$, representing DNAP, we see a linear distribution of chromosomes in the equatorial plane of the cell, which appears misaligned in the midleft region of the cell. This is a known characteristic effect of Taxol. ${ }^{50}$ The lipid component $\left(C_{3} \equiv C_{\mathrm{L}}\right)$ is localized at the cell membranes and lipid droplets. This factorization was using nine FSC $^{3}$ components, with the additional components corresponding to water, glass, and systematics given in the SI, Figure S32. The other Taxol treated cell showing circularly arranged $C_{\mathrm{D}}$ is shown in the SI, Figure S14.

Volumetric calculations over the chromosomal region and the whole cell as described previously were performed on this cell, and the results are given in Table 2 . The parameters of this calculation are given in the SI, Figure S20. Since Taxol arrests cells in metaphase, we compare the results of this factorization with the untreated prometaphase cell shown earlier. First, we note that the chromosomal and cell volumes of the Taxol

Table 2. Results of the Volumetric Calculations of $C_{1}, C_{2}$, and $C_{3}$ of Taxol Treated Cells ${ }^{a}$

\begin{tabular}{|c|c|c|c|c|c|}
\hline$i$ & type & $\gamma_{i}^{\mathrm{d}}$ & $V_{i}\left(\mu \mathrm{m}^{3}\right)$ & $V_{i} / V_{\mathrm{ROI}}(\%)$ & $M_{i}(\mathrm{pg})$ \\
\hline \multicolumn{6}{|c|}{ Figure 4 - Taxol - chromosomal volume - $1114 \mu \mathrm{m}^{3}(27 \%)$} \\
\hline 1 & $\mathrm{P}$ & 0.312 & $44.6 \pm 0.4$ & 4.00 & $58.0(66.8)$ \\
\hline 2 & $\mathrm{D}$ & 0.127 & $13 \pm 2$ & 1.15 & $19.2(10.4)$ \\
\hline 3 & $\mathrm{~L}$ & 0.753 & $19.2 \pm 0.2$ & 1.72 & 17.2 \\
\hline \multicolumn{6}{|c|}{ Figure 4 - Taxol - cell volume - $4174 \mu \mathrm{m}^{3}$} \\
\hline 1 & $\mathrm{P}$ & 0.312 & $191 \pm 2$ & 4.57 & $248.0(285.7)$ \\
\hline 2 & $\mathrm{D}$ & 0.127 & $32 \pm 2$ & 0.77 & $48.1(10.4)$ \\
\hline 3 & $\mathrm{~L}$ & 0.753 & $97 \pm 1$ & 2.32 & 86.7 \\
\hline \multicolumn{6}{|c|}{ Figure S14 - Taxol - chromosomal volume - $1001 \mu \mathrm{m}^{3}$ (19\%) } \\
\hline 1 & $\mathrm{P}$ & 0.312 & $43.8 \pm 0.4$ & 4.38 & $57.0(65.7)$ \\
\hline 2 & $\mathrm{D}$ & 0.127 & $12 \pm 2$ & 1.16 & $17.4(8.8)$ \\
\hline 3 & $\mathrm{~L}$ & 0.753 & $20.4 \pm 0.2$ & 2.04 & 18.3 \\
\hline \multicolumn{6}{|c|}{ Figure S14 - Taxol - cell volume $-5273 \mu \mathrm{m}^{3}$} \\
\hline 1 & $\mathrm{P}$ & 0.312 & $259 \pm 2$ & 4.92 & $337.0(388.2)$ \\
\hline 2 & $\mathrm{D}$ & 0.127 & $40 \pm 3$ & 0.76 & $60.0(8.8)$ \\
\hline 3 & $\mathrm{~L}$ & 0.753 & $146 \pm 2$ & 2.77 & 130.7 \\
\hline
\end{tabular}

${ }^{a^{a}}$ The rebalanced masses $\tilde{M}_{\mathrm{P}}$ and $\tilde{M}_{\mathrm{D}}$ are given in parentheses. treated cells we measured are similar to the prometaphase cell. Also, similar to the analysis for the labeled cells, here too we note a distribution of $C_{\mathrm{D}}$ outside the chromosomal volume. Therefore, we consider the rebalanced component masses. In the chromosomal region, the resulting $C_{\mathrm{D}}$ mass is consistent with the expected diploid DNA mass of $10-14 \mathrm{pg}$, while $C_{\mathrm{L}}$ has a mass approximately 6 times larger than in the prometaphase cell shown earlier. This could indicate that Taxol not only results in a misalignment of chromosomes, a known effect, but also in the accumulation of lipid vesicles in the chromosomal region. This is a plausible outcome of the drug treatment, because it is known that the organelles fragment and aggregate on the stabilized microtubule spindle. Generally, over the cell volumes, we observe a higher lipid mass in Taxol treated cells compared to the untreated samples. This is consistent with the known hypertriglyceridimic effect of Taxol and cis-platin based chemotherapy. ${ }^{51}$ Considering the protein component $C_{\mathrm{p}}$ over the cell volume, we note that its density (mass/cell volume) is double compared to the untreated prometaphase cell, in line with the hyperproteic effect of Taxol reported in literature. ${ }^{52}$

\section{CONCLUSIONS}

In this paper, we have demonstrated a method for unbiased quantitative chemically specific volumetric CARS microscopy on individual fixed U-2 OS cells and determined the intracellular dry masses of protein, DNAP, and lipid components. The attribution of proteins and DNAP chemical components was supported by comparison with correlative two-photon fluorescence imaging of Mitotracker-orange and DAPI staining. We investigated untreated cells during mitosis, and cells treated with the anticancer drug Taxol. The results are consistent with biomolecular changes expected during mitosis and the effects of drug treatment. In particular, cells treated with Taxol appear chemically similar to untreated samples, while containing a somewhat larger amount of proteins and lipid, a known effect of Taxol based chemotherapy. Moreover, the morphology of the nuclear material (DNAP) and its intracellular distribution in Taxol treated cells are characteristically different from those of control samples.

Generally, this work shows that CARS microscopy, traditionally utilized to study highly dense lipid aggregates, can provide quantitative chemical specificity and sensitivity in the detection of proteins and nuclear components in not lipid-rich cells where the imaged volume contains mostly water. Since CARS microscopy is a label-free tool applicable to unmodified samples, the progress shown here paves the way toward quantitative volumetric image analysis of proteins, lipids, and DNA in intact living cells. We note that while the PFA fixation used in the present work is known to have little influence on the cell mass and Raman spectra, as discussed in the SI, section 4 , the logical next step will be live cell imaging as it allows the dynamics of components to be measured, a longitudinal imaging providing a way through the heterogeneity. Future developments of the method, extending the spectral range to the fingerprint region, replacing $\mathrm{H}_{2} \mathrm{O}$ with $\mathrm{D}_{2} \mathrm{O}$ to reduce the spectral overlap, and combining CARS and SRS to optimize sensitivity, are expected to improve the errors in the extracted density distributions, to a level which allows to study cell metabolism and the cell cycle in even more detail, in a labelfree manner. Notably, such studies typically require the analysis of a large number of cells to gain statistical relevance, 
which provides a further challenge to the technology which is presently pursued. ${ }^{25}$ Following the proof-of-principle demonstration in the present work, focused studies answering specific biological questions, such as effect of topo II inhibition molecules on chemical changes in the nucleolus, could be commenced. Furthermore, applications of the demonstrated noninvasive quantitative volumetric chemical imaging, with picogram mass and submicrometer spatial resolution, are ubiquitous also in other fields, such as material science and pharmacology.

\section{ASSOCIATED CONTENT}

\section{S Supporting Information}

The Supporting Information is available free of charge on the ACS Publications website at DOI: 10.1021/acs.analchem.8b04706.

Additional information as referenced in the text and data corresponding to all figures shown (PDF)

\section{AUTHOR INFORMATION}

\section{Corresponding Authors}

*E-mail: erringtonrj@cardiff.ac.uk.

*E-mail: langbeinww@cardiff.ac.uk.

\section{ORCID}

Paola Borri: 0000-0002-7873-3314

Wolfgang Langbein: 0000-0001-9786-1023

\section{Present Address}

"Department of Imaging Physics, Delft University of Technology, Lorentzweg 1, 2628 CJ Delft, The Netherlands.

\section{Notes}

The authors declare no competing financial interest.

\section{ACKNOWLEDGMENTS}

The CARS microscope development was supported by the UK BBSRC Research Council (Grant No. BB/H006575/1). The FSC $^{3}$ data analysis development was supported by the UK EPSRC Research Council (Grant No. EP/H45848/1). A.K. acknowledges financial support by the Presidents Research Scholarship programme of Cardiff University. P.B. acknowledges the UK EPSRC Research Council for her Leadership fellowship award (Grant No. EP/I005072/1). W.L. acknowledges support by a Leverhulme Royal Society Research Fellowship (Grant No. LT20085). The authors thank Iestyn Pope for assistance in the data acquisition.

\section{REFERENCES}

(1) Rubart, M. Circ. Res. 2004, 95, 1154-1166.

(2) Becker, W. J. Microsc. 2012, 247, 119-136.

(3) Chen, B.-C.; Legant, W. R.; Wang, K.; Shao, L.; Milkie, D. E.; Davidson, M. W.; Janetopoulos, C.; Wu, X. S.; Hammer, J. A.; Liu, Z. Science 2014, 346, 1257998.

(4) Corriden, R.; Insel, P.; Junger, W. G. Am. J. Physiol. cell physiology 2007, 293, 1420-1425.

(5) Piredda, P.; Berning, M.; Boukamp, P.; Volkmer, A. Anal. Chem. 2015, 87, 6778-6785.

(6) Müller, W.; Kielhorn, M.; Schmitt, M.; Popp, J.; Heintzmann, R. Optica 2016, 3, 452-457.

(7) Zumbusch, A.; Langbein, W.; Borri, P. Prog. Lipid Res. 2013, 52, 615-632.

(8) Evans, C. L.; Xie, X. S. Annu. Rev. Anal. Chem. 2008, 1, 883-909.

(9) Freudiger, C. W.; Min, W.; Saar, B. G.; Lu, S.; Holtom, G. R.; He, C.; Tsai, J. C.; Kang, J. X.; Xie, X. S. Science 2008, 322, 1857.
(10) Day, J. P. R.; Domke, K. F.; Rago, G.; Kano, H.; Hamaguchi, H.-o.; Vartiainen, E. M.; Bonn, M. J. Phys. Chem. B 2011, 115, 77137725.

(11) Cheng, J.-X.; Xie, X. S. Science 2015, 350, 350.

(12) Camp, C. H.; Cicerone, M. T. Nat. Photonics 2015, 9, 295305.

(13) Karuna, A.; Masia, F.; Borri, P.; Langbein, W. J. Raman Spectrosc. 2016, 47, 1167-1173.

(14) Cicerone, M. T.; Aamer, K. A.; Lee, Y. J.; Vartiainen, E. J. Raman Spectrosc. 2012, 43, 637-643.

(15) Liu, Y.; Lee, Y. J.; Cicerone, M. T. Opt. Lett. 2009, 34, 1363.

(16) Rinia, H. A.; Bonn, M.; Müller, M.; Vartiainen, E. M. ChemPhysChem 2007, 8, 279-287.

(17) Masia, F.; Glen, A.; Stephens, P.; Borri, P.; Langbein, W. Anal. Chem. 2013, 85, 10820-10828.

(18) Lee, Y. J.; Moon, D.; Migler, K. B.; Cicerone, M. T. Anal. Chem. 2011, 83, 2733-2739.

(19) Ozeki, Y.; Umemura, W.; Otsuka, Y.; Satoh, S.; Hashimoto, H.; Sumimura, K.; Nishizawa, N.; Fukui, K.; Itoh, K. Nat. Photonics 2012, $6,845-851$.

(20) Zhang, D.; Wang, P.; Slipchenko, M. N.; Ben-Amotz, D.; Weiner, A. M.; Cheng, J.-X. Anal. Chem. 2013, 85, 98-106.

(21) Masia, F.; Karuna, A.; Borri, P.; Langbein, W. J. Raman Spectrosc. 2015, 46, 727-734.

(22) Di Napoli, C.; Pope, I.; Masia, F.; Watson, P.; Langbein, W.; Borri, P. Biomed. Opt. Express 2014, 5, 1378-1390.

(23) Di Napoli, C.; Pope, I.; Masia, F.; Langbein, W.; Watson, P.; Borri, P. Anal. Chem. 2016, 88, 3677.

(24) Masia, F.; Glen, A.; Stephens, P.; Langbein, W.; Borri, P. J. Biophot. 2018, 11, No. e201700219.

(25) Masia, F.; Pope, I.; Watson, P.; Langbein, W.; Borri, P. Anal. Chem. 2018, 90, 3775-3785.

(26) Uhlmann, F. Curr. Opin. Cell Biol. 2001, 13, 754-761.

(27) Ouellet, J.; Barral, Y. J. Cell Biol. 2012, 196, 305-313.

(28) Boucrot, E.; Kirchhausen, T. PLoS One 2008, 3, e1477.

(29) Mora-Bermudez, F.; Ellenberg, J. Methods 2007, 41, 158-167.

(30) Nebenfuhr, A. Cell Division Control in Plants. In Plant Cell Monographs; Verma, D. S., Hong, Z., Eds.; Springer: Berlin, 2007; Vol. 9, pp 195-206.

(31) Imoto, Y.; Yoshida, Y.; Yagisawa, F.; Kuroiwa, H.; Kuroiwa, T. Microscopy 2011, 60, S117-S136.

(32) Neumann, B.; Walter, T.; Hériché, J.-K.; Bulkescher, J.; Erfle, H.; Conrad, C.; Rogers, P.; Poser, I.; Held, M.; Liebel, U.; et al. Nature 2010, 464, 721-727.

(33) Popescu, G.; Park, Y.; Lue, N.; Best-Popescu, C.; Deflores, L.; Dasari, R. R.; Feld, M. S.; Badizadegan, K. Am. J. Physiol. Cell Physiol. 2008, 295, C538-C544.

(34) Mir, M.; Wang, Z.; Shen, Z.; Bednarz, M.; Bashir, R.; Golding, I.; Prasanth, S. G.; Popescu, G. Proc. Natl. Acad. Sci. U. S. A. 2011 108, 13124-13129.

(35) Zangle, T. A.; Teitell, M. A. Nat. Methods 2014, 11, 12211228.

(36) Matson, D. R.; Stukenberg, P. Mol. Interventions 2011, 11, 141150.

(37) Cottet-Rousselle, C.; Ronot, X.; Leverve, X.; Mayol, J.-F. Cytometry, Part A 2011, 79A, 405-425.

(38) Kapuscinski, J. Biotech. Histochem. 1995, 70, 220-233.

(39) Pope, I.; Langbein, W.; Watson, P.; Borri, P. Opt. Express 2013, 21, 7096-7106.

(40) The data presented in this work are available from the Cardiff University data archive, DOI: 10.17035/d.2019.0066725262.

(41) Rustan, A. C.; Drevon, C. A. eLS; John Wiley \& Sons, Ltd, 2001; Chapter Fatty Acids: Structures and Properties.

(42) Otaki, J. M.; Tsutsumi, M.; Gotoh, T.; Yamamoto, H. J. Chem. Inf. Model. 2010, 50, 690-700.

(43) Colland, Y.; Kosma, M. V. In Cancer management in man; Goldson, A. L., Ed.; Kluwer Academic Publishers: 1989; Chapter 10, p 135. 
(44) Database, P. C. Palmitic acid. https://pubchem.ncbi.nlm.nih. gov/compound/985 (accessed Mar. 16, 2016).

(45) Database, P. C. Stearic Acid. Online, https://pubchem.ncbi. nlm.nih.gov/compound/5281 (accessed Mar. 16, 2016).

(46) Database, P. C. Oleic acid. Online, https://pubchem.ncbi.nlm. nih.gov/compound/445639\#section=Solubility (accessed Jul. 11 2018).

(47) Milo, R.; Phillips, R. Cell biology by the numbers, 1st ed.; Garland Science, 2015; p 72.

(48) Brito, D.; Rieder, C. L. Cell Motil. Cytoskeleton 2009, 66, 437447.

(49) Yvon, A.-M. C.; Wadsworth, P.; Jordan, M. Mol. Biol. Cell 1999, 10, 947-959.

(50) Jordan, M. A.; Wilson, L. Nat. Rev. Cancer 2004, 4, 253-265.

(51) Wang, G.; Su, C.; Yin, T. Mol. Clin. Oncol. 2017, 6, 261-265.

(52) Derry, W. B.; Wilson, L.; Jordan, M. A. Biochemistry 1995, 34, 2203-2211. 